\title{
Transient Pseudohypoaldosteronism in a 5-Month-old Infant Manifested as a Failure to Thrive
}

\author{
Jung Won Lee, Su Jin Cho, Hae Soon Kim \\ Department of Pediatrics, Ewha Womans University College of Medicine, Seoul, Korea
}

Pseudohypoaldosteronism (PHA) in infants is manifested by presence of hyperkalemia, hyponatremia, and metabolic acidosis. At initial stages, PAH is generally suspected as congenital adrenal hyperplasia. Transient PHA has been reported in infants with urinary tract infection and urinary tract malformation. We report a case of 5-month-old infant with failure to thrive and finally diagnosed with transient PHA due to urinary tract infection with vesicoureteral reflux. (Ewha Med J 2019;42(1):6-9)

\section{Introduction}

Pseudohypoaldosteronism (PHA) comprises of a heterogeneous group of disorders of electrolyte metabolism characterized by an apparent state of renal tubular unresponsiveness or resistance to the action of aldosterone. It is manifested by presence of salt wasting, hyperkalemia, and metabolic acidosis [1]. Signs and symptoms of PHA can be rather unspecific and include poor feeding, poor weight gain or failure to thrive, vomiting, diarrhea, polyuria, and dehydration [2]. PHA is classified as primary and secondary type. Secondary transient PHA has been reported in infants with urinary tract infection (UTI) and urinary tract malformation. The acute biochemical presentation of PHA is identical to adrenal crisis due to congenital adrenal hy-
Received September 4, 2018

Revised October 16, 2018

Accepted October 16, 2018

Corresponding author

Hae Soon Kim

Department of Pediatrics, Ewha Womans University College of Medicine, 1071

Anyangcheon-ro, Yangcheon-gu, Seoul 07985, Korea

Tel: 82-2-2650-5569, Fax: 82-2-2653-3718

E-mail: hyesk@ewha.ac.kr

Key Words

Pseudohypoaldosteronism; Failure to thrive; Urinary tract infections; Vesico-ureteral reflux

This is an Open Access article distributed under the terms of the Creative Commons Attribution Non-Commercial License (http://creativecommons.org/licenses/by-nc/4.0) which permits unrestricted non-commercial use, distribution, and reproduction in any medium, provided the original work is properly cited. 
been breastfeeding and weighed $5 \mathrm{~kg}$ at 3 months of age but developed no weight gain after that.

On physical examination, she was afebrile and had not so ill looking appearance. Body temperature was $36.9^{\circ} \mathrm{C}$, pulse rate 120 beats $/ \mathrm{min}$. respiration rate 40 beats $/ \mathrm{min}$, and blood pressure was $85 / 60 \mathrm{mmHg}$. Virilization of external genitalia or pigmentation were not remarkable. Initial serum sodium was 125 $\mathrm{mEq} / \mathrm{L}$ and serum potassium was $6.1 \mathrm{mEq} / \mathrm{L}$. The serum CRP level was $4.08 \mathrm{mg} / \mathrm{dL}$ and serum ESR level was $60 \mathrm{~mm} / \mathrm{hr}$. Urine analysis revealed pyuria. Intravenous saline and antibiotics were started after examination of urine culture. Catheterized urine culture was positive for Serratia marcescens. The initial serum 17-hydroxyprogesterone level was $0.73 \mathrm{ng} / \mathrm{mL}$ (normal range, 0.13 to $0.106 \mathrm{ng} / \mathrm{mL}$ ) and aldosterone level was markedly elevated $(17,800 \mathrm{pg} / \mathrm{mL}$ ) (normal range, 50 to $900 \mathrm{pg} / \mathrm{mL}$ ), and urinary sodium was $30 \mathrm{mg} / \mathrm{L}$. The changes in clinical parameters led to apparent diagnosis of PHA. The infant's serum sodium and potassium levels were normalized after 48 hours of intravenous sodium replacement and antibiotics therapy, and inflammatory markers were normalized. The renal ultrasonography showed mild atrophy of right kidney and compensatory hypertrophy of left kidney. Technetium-99m-dimercaptosuccinic acid renal scan demonstrated multiple renal cortical defects in both kidneys and generalized areas of diminished uptake in right kidney (Fig. 1). The voiding cystourethrography showed right-sided grade $\mathrm{V}$ and left-sided grade IV vesicoureteral reflux (Fig. 2). The follow-up electrolyte levels remained within normal range and aldosterone level was decreased without oral sodium replacement and proper weight gain was achieved during outpatient follow-up.

\section{Discussion}

A variety of factors contribute to the pathogenesis of hyponatremia and hyperkalemia in neonatal and infant periods. In these children is very important to establish or exclude $\mathrm{CAH}$ because this is a life-threatening condition [3]. More than 90\% of $\mathrm{CAH}$ are caused by 21-hydroxylase deficiency (so-called classical salt wasting form). The clinical presentation of 21-hydroxylase deficiency in female results in masculinized external genitalia and vomiting within a few days/weeks after birth, severe renal wasting, and dehydration in both sexes. Our patient was an infant with poor weight gain and with normal external genitalia. He showed hyponatremia, hyperkalemia and elevated serum aldosterone level.

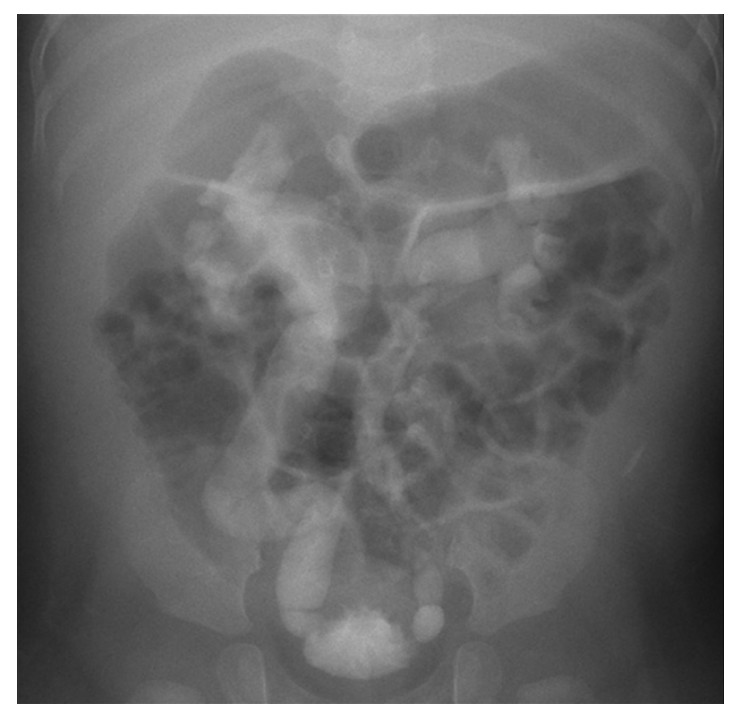

Fig. 2. Voiding cystourethrography findings show vesicoureteral reflux (right, grade V; left, grade IV).

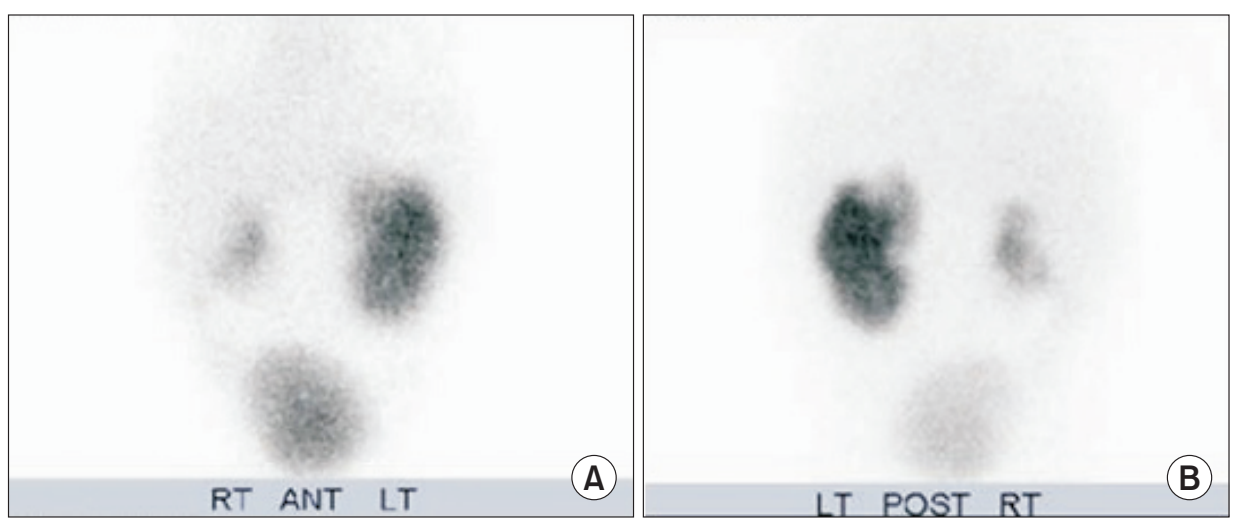

Fig. 1. The technetium-99m-dimercaptosuccinic acid (DMSA) renal scan findings show multiple cortical defects in both the kidneys and generalized areas of diminished uptake in right kidney. (A) Anterior view of DMSA. (B) Posterior view of DMSA. RT, right; ANT, anterior; LT, left; POST, posterior. 
PHA is subdivided into primary (genetic) and secondary (or transient) forms. Primary PHA has two clinically and genetically distinct forms with either an autosomal recessive or an autosomal dominant disorder [4]. The autosomal recessive form (the so-called multiple organ form) is associated with mutation in the subunit of the epithelial sodium channel ( $\mathrm{ENaC}$ gene), causing salt wasting not only from the kidney, but also from the colon, sweat glands, and salivary glands $[1,5]$. The autosomal dominant form is due to mutation in the mineralocorticoid receptor gene, which prevents normal receptor function and, hence, causes renal salt wasting [6].

A secondary form of PHA, limited to the kidney, may be associated either with medications or tubulointerstitial disease. Secondary PHA has been described most frequently in neonates and young infants in association with UTI and/or urinary tract malformations such as, hydronephrosis, ureteropelvic junction obstruction, vesicoureteral reflux, or posterior urethral valve $[7,8]$. After treatment for UTI and/or surgical therapy, disappearance of secondary aldosterone resistance has been noted.

Rodriguez-Soriano et al. [7] first described the entity of transient PHA to be associated with obstructive uropathy in 1983. The mechanism is poorly understood, but it is speculated that it may be due to parenchymal scarring secondary to obstruction and tubular aldosterone resistance secondary to endotoxin damage of the aldosterone receptors from cytokine such as, TGF- $\beta$ [9]. Early infancy, obstructive uropathy, and UTI are three major risk factors for the development of secondary PHA. Most of the patients are younger than 3 months of age, which suggests that immature renal tubules may be responsible for the development of resistance to aldosterone. Furthermore, the severity of salt wasting is inversely correlated with age, thus supporting a role for tubular immaturity [10]. Bogdanovic et al. [2] reported that $90 \%$ of patients with transient PHA were less than 3 months of age and $89 \%$ of them suffered from urinary tract malformation associated with UTI. Melzi et al. [11] never observed transient PHA in infants older than 3 months despite the presence of urinary tract malformation.

Bowden et al. [12] first found that autosomal dominant PHA with a mineralocorticoid receptor gene (NR3C2) mutation, presenting with salt wasting crisis was associated with UTI and posterior urethral valves, thereby mimicking secondary PHA [13]. If serum aldosterone is persistently elevated after UTI treatment, then NR3C2 gene testing should be considered to identify the cause, to determine future recurrence risk, and to prevent serious salt wasting in a subsequent family member. It should be noted that our patient was 5-month-old infant and only manifested poor weight gain and failure to thrive since 3 months of age. The differentiation between $\mathrm{CAH}$ and PHA is difficult because the electrolyte imbalances are identical, however virilization or pigmentation is not found in PHA. Delayed antibiotics treatment in patients with UTI misdiagnosed as $\mathrm{CAH}$ could be fatal because of the life-threatening risk of septic shock. Therefore, it is mandatory to include urinalysis and $a b-$ domen ultrasound in work-up of patients with salt wasting to exclude infection or urinary tract malformation [14].

Transient PHA should be suspected in an infant with severe hyponatremia, hyperkalemia and failure to thrive without virilization or pigmentation. Urinalysis and urinary tract imaging studies should always be performed in order to allow early diagnosis of transient PHA, thus preventing serious complications and inappropriate treatments.

\section{References}

1. Geller DS. Mineralocorticoid resistance. Clin Endocrinol (Oxf) 2005;62:513-520.

2. Bogdanovic R, Stajic N, Putnik J, Paripovic A. Transient type 1 pseudo-hypoaldosteronism: report on an eight-patient series and literature review. Pediatr Nephrol 2009;24:2167-2175.

3. Dolezel Z, Starha J, Novotna D, Dostalkova D. Secondary pseudohypoaldosteronism in an infant with pyelonephritis. Bratisl Lek Listy 2004;105:435-437.

4. Hanukoglu A. Type I pseudohypoaldosteronism includes two clinically and genetically distinct entities with either renal or multiple target organ defects. J Clin Endocrinol Metab 1991;73:936-944.

5. Zennaro MC, Lombes M. Mineralocorticoid resistance. Trends Endocrinol Metab 2004;15:264-270.

6. Pujo L, Fagart J, Gary F, Papadimitriou DT, Claes A, Jeunemaitre $\mathrm{X}$, et al. Mineralocorticoid receptor mutations are the principal cause of renal type 1 pseudohypoaldosteronism. Hum Mutat 2007;28:33-40.

7. Rodriguez-Soriano J, Vallo A, Oliveros R, Castillo G. Transient pseudohypoaldosteronism secondary to obstructive uropathy in infancy. J Pediatr 1983;103:375-380.

8. Proctor G, Linas S. Type 2 pseudohypoaldosteronism: new insights into renal potassium, sodium, and chloride handling. Am J Kidney Dis 2006;48:674-693.

9. Watanabe T. Reversible secondary pseudohypoaldosteronism. Pediatr Nephrol 2003;18:486.

10. Belot A, Ranchin B, Fichtner C, Pujo L, Rossier BC, Liutkus A, 
et al. Pseudohypoaldosteronisms, report on a 10-patient series. Nephrol Dial Transplant 2008;23:1636-1641.

11. Melzi ML, Guez S, Sersale G, Terzi F, Secco E, Marra G, et al. Acute pyelonephritis as a cause of hyponatremia/hyperkalemia in young infants with urinary tract malformations. Pediatr Infect Dis J 1995; 14:56-59.

12. Bowden SA, Cozzi C, Hickey SE, Thrush DL, Astbury C, Nuthakki S. Autosomal dominant pseudohypoaldosteronism type 1 in an infant with salt wasting crisis associated with urinary tract infection and obstructive uropathy. Case Rep Endocrinol 2013;2013:524647.

13. Fujinaga S, Ohtomo Y, Someya T, Shimizu T, Yamashiro Y. Transient pseudohypoaldosteronism complicating acute renal failure in an infant with vesico-ureteral reflux and pyelonephritis. Pediatr Int 2009;51:744-746.

14. Delhikumar CG, Narayanan P, Mahadevan S. Pseudohypoaldosteronism masquerading as congenital adrenal hyperplasia. Indian J Pediatr 2012;79:115-116. 